\title{
Maternal factors regulate stress responsiveness in the neonatal rat
}

\author{
FRANCESCA CIRULLI \\ Laboratorio di Fisiopatologia di Organo e di Sistema, Istituto Superiore di Sanitá, Rome, Italy \\ and Stanford University School of Medicine, Stanford, California \\ and \\ SAMI L. GOTTLIEB, PATRICIA ROSENFELD, and SEYMOUR LEVINE \\ Stanford University School of Medicine, Stanford, California
}

\begin{abstract}
Experiment 1 compared (basal and stress-induced) adrenocortical secretion in infants individually isolated for a 24-h period with infants deprived of their mothers but in contact with their littermates. Maternal deprivation resulted in the activation of the pups' hypothalamic-pituitaryadrenal (HPA) system, independent of the social contact with the littermates. Results from Experiment 2 showed that nonnutritional contact with a thelectomized (nipple-removed) dam was only partially effective in suppressing the HPA system activity, and this effect was age dependent. Experiment 3 explored the possibility that glucocorticoids transported through the milk might be involved in the inhibition of the HPA system. Infants that remained with an adrenalec. tomized dam for $24 \mathrm{~h}$ prior to testing did not differ from their nondeprived counterparts on Day 8 but did differ on Day 12, suggesting that, at the older ages, the nutritional demands were greater and that the effects observed were probably due to undernutrition.
\end{abstract}

The neonatal rat shows a markedly diminished capacity to elicit the well-described cascade of hormonal responses that result in a marked elevation of corticotropin (ACTH) and glucocorticoid (GC) secretion following stress. Thus, between Postnatal Days 4 and 14, the neonate shows litthe, if any, secretion of these hormones when exposed to a wide variety of stimuli that would reliably activate their secretion in the adult. This period has become known as the stress hyporesponsive period (SHRP; for reviews, see De Kloet, Rosenfeld, Van Eekelen, Sutanto, \& Levine, 1988; Sapolsky \& Meaney, 1986).

The mechanisms responsible for the SHRP have not yet been elucidated. It has been hypothesized that an enhanced negative feedback action of corticosterone (CORT)-the main GC in the rat-may underlie the observed hyporesponsiveness of the system during this period (Sakly \& Koch, 1983; Schapiro, 1965; Walker, Sapolsky, Meaney, Vale, \& Rivier, 1986; but see Widmaier, 1990). Recent reports suggest that the mother-infant relationship may constitute an important factor in the regulation of the hypothalamic-pituitary-adrenal (HPA) system during de-

\footnotetext{
The research was supported by Grant HD-02881 from National Institute of Child Health and Human Development, Grant MH-45006 from the National Institute of Mental Health (NIMH), and U.S. Public Health Service Research Scientist Award MH-19936 from NIMH to S. Levine. F. Cirulli was partially supported by the CNR-FATMA "stress" subproject, and by CNR grant 890410304 . We thank Enrico Alleva for the helpful comments on the manuscript, Helen Hu for carrying out the corticosterone assays, and Sandra Wiener for her assistance in analyzing the data. Correspondence should be addressed to F. Cirulli, Laboratory of Developmental Psychobiology, Building 7-930T, Department of Psychiatry and Behavioral Sciences, Stanford University School of Medicine, Stanford University, Stanford, CA 94305-5095.
}

velopment. Thus, infant rats deprived of their mothers for $24 \mathrm{~h}$ show significantly greater plasma levels of CORT following exposure to novelty, saline injection, or exogenous administration of ACTH than do nondeprived infants (Levine, Huchton, Wiener, \& Rosenfeld, 1991; Rosenfeld et al., 1991; Stanton, Gutierrez, \& Levine, 1988).

Maternal factors appear to influence the neonatal HPA system in at least two different ways. First, previous studies have shown that the presence of the mother is sufficient to suppress the activation of the infant's HPA system in situations that elicit a rise in CORT secretion. In this case, contact per se in the absence of feeding appears to be the critical maternal variable responsible for this suppression (Stanton \& Levine, 1988; Stanton, Wallstrom, \& Levine, 1987). Second, factors of maternal origin appear to be at least partly responsible for maintaining the overall hyporesponsiveness of the infant's HPA system. Thus, until approximately Postnatal Day 14, the neonatal rat exhibits an adrenocortical response to a variety of stimuli that is very small in magnitude, unless it has previously undergone a prolonged period of maternal deprivation (e.g., $24 \mathrm{~h}$ ) immediately prior to the stimulation. It should be noted that although the presence of the mother may have an acute effect in preventing the response to an immediate challenge, prolonged isolation (longer than $8 \mathrm{~h}$ ) is required before disinhibition of the infant's HPA system occurs (Levine et al., 1991; Kuhn, Pauk, \& Schanberg, 1990). Although Kuhn et al. (1990) report that levels of CORT are significantly elevated following $2 \mathrm{~h}$ of separation, the absolute levels observed at this time correspond approximately to $1 \mu \mathrm{g} / \mathrm{dl}$. This is markedly lower than the level seen after $24 \mathrm{~h}$ of deprivation and is statis- 
tically significant because of the exceptionally low basal levels obtained by these investigators. These early changes were not replicated in the Levine et al. (1991) study, which examined the time course of the changes in HPA activity as a function of maternal deprivation.

A number of factors might be involved in the regulation of the HPA axis activity during the deprivation interval: absence of littermates, maternal contact, suckling, feeding, or transmission of maternal hormones through the milk. The present studies were undertaken to explore the effects of several of these possible variables on the activity of the HPA system. In particular, we focused on the effects of social companions and of nonnutritional maternal contact, as well as the influence of CORT transmitted through milk on both basal levels and stressinduced changes in CORT.

\section{GENERAL METHODS}

\begin{abstract}
Animals
The subjects were 8-, 12-, and 16-day-old rat pups bred from Sprague-Dawley females (Holtzmann, Madison, WI) and LongEvans males (Simonsen, Gilroy, CA). Age was determined by checking for births every day at approximately 0830 and $1630 \mathrm{~h}$; the day of birth was designated as Day 0 . Litters were housed with their mothers in transparent polycarbonate cages (Nalgene, $48.5 \times$ $25.6 \times 19.0 \mathrm{~cm}$ ) with approximately $3 \mathrm{~cm}$ of sawdust shavings on the cage floor.

On the first day after birth (Day 1) litters were culled to 8 healthy animals (usually 4 females and 4 males) and placed in a clean cage. From this moment on, the animals were not handled in any way, nor were their cages cleaned, until the time of deprivation or testing. Rat chow (Wayne Rodent Blox, Allied Mills, Chicago, IL) and tap water were provided ad lib. The laboratory was maintained under constant temperature $\left(22^{\circ} \mathrm{C}\right)$ and lighting $(12: 12$-h light:dark cycle) conditions. The animals were maternally deprived and tested 4-8 $\mathrm{h}$ after light onset.
\end{abstract}

\section{Equipment}

Pups deprived individually (see Experiment 1) were placed in one of the individual compartments of a deprivation box. This consisted of a $37 \times 29 \times 13 \mathrm{~cm}$ box made of $0.64-\mathrm{cm}$ clear Plexiglas. The chamber was divided into 12 individual compartments $(8 \times 9 \times$ $13 \mathrm{~cm})$. Each compartment was ventilated through four $1-\mathrm{cm}$ diameter holes in the roof and was filled with approximately $5 \mathrm{~cm}$ of sawdust shavings taken from the home cage. This allowed the pups to partially regulate their body temperatures by burrowing for warmth or returning to the surface for cooling. Compartments could be accessed through a sliding Plexiglas roof. The chamber was placed on an electric heating pad (General Electric) set at $30^{\circ}-33^{\circ} \mathrm{C}$, in a room adjacent to the main colony room, and was illuminated on the same light:dark cycle. The deprivation box was used only in Experiment 1. Once it was found that pups deprived of the mother together with their siblings $(\mathrm{M}-\mathrm{L}+)$ or individually deprived $(M-L-)$ did not differ, they were subsequently deprived with their littermates $(\mathbf{M}-\mathrm{L}+)$.

The novelty chambers consisted of small $(28 \times 17 \times 13 \mathrm{~cm})$ polyethylene rodent cages. The chambers were placed on an electric heating pad (General Electric) set at $30^{\circ}-33^{\circ} \mathrm{C}$, in a room adjacent to the main colony room. Clean paper towels were placed on the floor of each chamber at the beginning of each testing session.

\section{Procedure}

Deprivation. Nondeprived pups $(\mathrm{M}+\mathrm{L}+)$ were left undisturbed with their mothers until the time of testing. Pups in the M-L+con- dition were deprived with their littermates (i.e., $24 \mathrm{~h}$ prior to testing), the mother was removed from the home cage, and the cage was subsequently placed on a heating pad (General Electric) set at $30^{\circ}-33^{\circ} \mathrm{C}$, in a room adjacent to the main colony room (illuminated on the same light:dark cycle). Individually deprived (M-L-) pups were separated from their mothers and littermates $24 \mathrm{~h}$ prior to testing and were placed in individual compartments of the deprivation box.

Blood collection. Trunk blood was collected from all animals in heparinized centrifuge vials and centrifuged at $2,000 \mathrm{rpm}$ for $20 \mathrm{~min}$. Plasma was extracted and frozen until subsequent CORT radioimmunoassay. This assay was a modification of the cortisol radioimmunoassay of Klemm and Gupta (1975); a specific antibody for CORT (B3-316, Endocrine Sciences, Tarzana, CA) was used.

\section{Data Analysis}

The results were analyzed by analysis of variance (ANOVA) procedures, with the level of significance set at $p<.05$ (Winer, 1971). Where appropriate, tests for simple main and interaction effects and subsequent post hoc comparisons were made by Newman-Keuls procedures.

\section{EXPERIMENT 1}

In previous deprivation studies, the pups were separated from their mothers and placed in individual heated compartments prior to being exposed to various stimuli (Rosenfeld et al., 1991; Stanton et al., 1988). These infants were therefore not only maternally deprived, but also socially isolated. Neonatal rats show a strong tendency to maintain close proximity to their littermates; they do this predominantly by huddling (Alberts, 1978). Total isolation of a pup from the dam and its littermates elicits high levels of ultrasonic vocalizations, often called distress vocalizations, accompanied by increases in locomotion and self-grooming (Hofer \& Shair, 1978). Social contact, either with a lactating female or with a littermate, reduces these behavioral responses (Hofer, 1987; Hofer \& Shair, 1980). Thus, it is not clear whether the crucial element in the regulation of the HPA axis is the presence of the mother and/or the litter.

In the first of three series of experiments to be reported, we examined the CORT response following exposure to novelty in 24-h maternally-deprived 8-, 12-, and 16-dayold rat pups that were either socially isolated or left with their littermates during the deprivation period. These animals were compared with pups that remained with their mother (i.e., nondeprived) until testing time.

\section{Method}

\section{Procedure}

Deprivation. Pups were taken from 6-9 litters at each age. Litters in each age group were randomly assigned to one of three pretesting conditions: nondeprived $(M+L+)$, deprived with their littermates $(M-L+)$, or individually deprived $(M-L-)$.

Testing. On the day of testing (i.e., Postnatal Days 8, 12, and 16), each of the litters in each deprivation condition and age group underwent equivalent testing procedures. From the 8 pups in each litter, 4 ( 2 males and 2 females) were weighed and then bloodsampled immediately (nontreated, or $N T$ ). The remaining 4 pups were then weighed, placed individually in one of the novelty test 

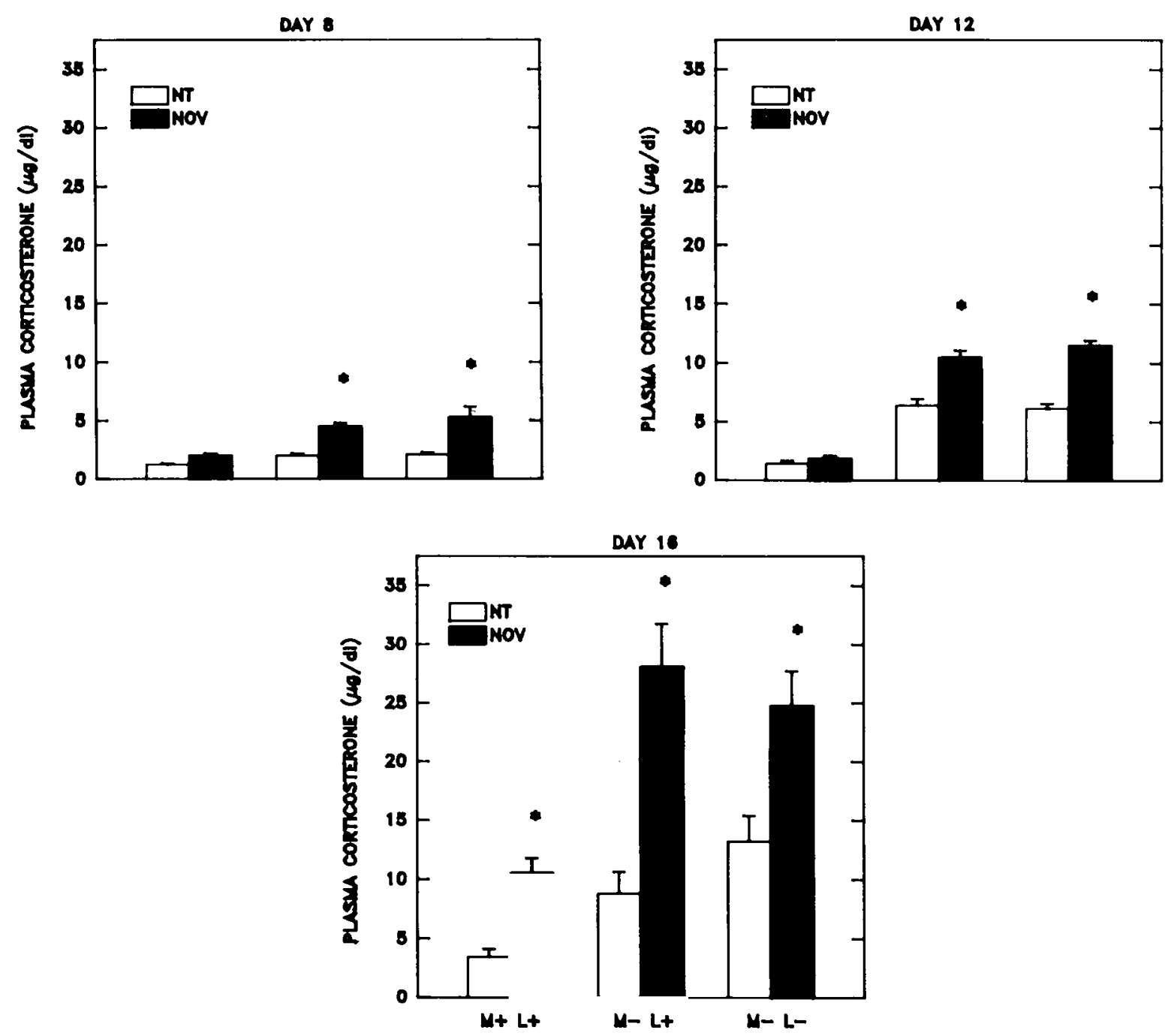

Figure 1. Plasma corticosterone levels $(\mu \mathrm{g} / \mathrm{dl})$ in rat pups at different ages, under the sampling conditions of Experiment 1 . During the $24 \mathrm{~h}$ prior to testing, pups were left with their mothers $(\mathbf{M}+\mathbf{L}+)$, maternally deprived with their littermates $(M-L+)$, or individually deprived (M-L-). At the time of testing, they were either sacrificed immediately (nontreated, or NT) or placed individually in a novelty test chamber for 30 min and then blood-sampled at the end of this period (novelty, or NOV). Values correspond to the mean $( \pm S E M$ ). Within each group, values statistically greater than NT are marked with an asterisk. For intergroup post hoc comparisons, refer to the Results section in the text.

chambers for $30 \mathrm{~min}$, and blood-sampled at the end of this period (novelty, or $N O V$ ).

\section{Results}

The results of Experiment 1 are shown in Figure 1. Data were analyzed separately at each age by means of a 3 (condition, $\mathrm{M}+\mathrm{L}+\mathrm{vs}$. $\mathrm{M}-\mathrm{L}+$ vs. $\mathrm{M}-\mathrm{L}-$ ) $\times 2$ (treatment, NT vs. NOV) $\times 2$ (sex, male vs. female) ANOVA. Once it was determined that sex was not a significant factor, the data were collapsed across this variable and analyzed by means of a 3 (condition) $\times 2$ (treatment) ANOVA. Interactions between condition and treatment were found to be due to litter differences $(p<.05)$; individual values from each litter were therefore averaged, resulting in a single
NT value and a single NOV value from each litter $(n=$ 6-9 for each cell).

\section{Postnatal Day 8}

An ANOVA revealed an interaction between condition and treatment $[F(2,30)=4.98, p<.05]$. A NewmanKeuls analysis of this interaction indicated that NT levels of plasma CORT did not differ across conditions. Only the deprived groups (i.e., $\mathrm{M}-\mathrm{L}-$ and $\mathrm{M}-\mathrm{L}+$ ) showed a significant elevation in CORT levels above NT values following exposure to novelty. There was no significant difference between the $\mathrm{M}-\mathrm{L}-$ and $\mathrm{M}-\mathrm{L}+$ conditions. Thus, Postnatal Day 8 pups can show an adrenocortical response to novelty exposure, but they require prior maternal dep- 
rivation in order to do so. Both deprivation procedures were equally capable of eliciting such an effect.

\section{Postnatal Day 12}

An ANOVA revealed an interaction between condition and treatment $[F(2,34)=16.28, p<.001]$. A NewmanKeuls analysis of this interaction indicated that CORT levels in $\mathbf{M}-\mathbf{L}-$ and $\mathbf{M}-\mathbf{L}+$ pups differed significantly from those of their $\mathrm{M}+\mathrm{L}+$ counterparts both at rest (NT) and following exposure to novelty (NOV). NOV CORT levels of both deprived groups were significantly elevated over base, and they did not differ from each other. At this age, therefore, maternal deprivation significantly increases CORT secretion both at rest and following exposure to novelty.

\section{Postnatal Day 16}

An ANOVA showed an interaction between condition and treatment $[F(2,32)=3.95, p<.05]$. Post hoc analysis of group differences showed that NT CORT levels in $M-L+$ pups were significantly greater than those found in $\mathrm{M}+\mathrm{L}+$ pups. However, in contrast to what was observed in 12-day-old pups, resting CORT levels of M-Lpups did not differ from those found in $M+L+$ animals. Once again, when exposed to novelty, pups in both deprived conditions did not differ from each other, and they showed significantly higher CORT levels than $M+L+$ animals. The increase in CORT levels observed in $\mathrm{M}+\mathrm{L}+$ pups exposed to novelty suggests that, by this age, prior maternal deprivation is not required in order to respond to a psychological stimulus. However, if pups have been maternally deprived, regardless of whether or not they have remained in the presence of littermates, the intensity of the response is markedly increased.

Taken as a whole, the results of Experiment 1 indicate that maternal deprivation, and not isolation from the littermates, underlies the observed changes in the HPA system. In addition, the data confirm the findings of Stanton et al. (1988), which suggest that maternal deprivation potentiates the CORT response to novelty, and that this potentiation is superimposed on an age-dependent general increase in HPA responsiveness.

\section{EXPERIMENT 2}

In Experiment 1, we demonstrated that the reduced activity of the neonatal HPA system is in some way related to the presence of the mother. One of the interesting features of maternal regulation is that different aspects of maternal behavior appear to regulate specific physiological processes in the infant. Thus, for example, following $18 \mathrm{~h}$ of maternal deprivation, 14-day-old rat pups show a $40 \%$ decrease in cardiac rate. This decrease appears to be a consequence of nutritional deprivation, since it can be reversed by intragastric milk infusion (Hofer, 1973). Maternal deprivation also alters the infant's sleep- wake cycles; in this case, however, the periodicity of milk delivery per se (rather than nutrition) appears to be the critical maternal variable (Hofer \& Shair, 1982). Finally, maternal deprivation leads to a sharp decline in the secretion of growth hormone and ornithine decarboxylase (a growth-related enzyme). The secretion of these compounds can be normalized by stroking the maternally deprived pup with a brush (Evoniuk, Kuhn, \& Schanberg, 1979; Kuhn, Butler, \& Schanberg, 1978). This, presumably, would mimic the tactile stimulation that is normally provided by the mother in order to induce urination and defecation in the pup.

The specific aspect of maternal behavior responsible for the regulation of the HPA system remains unknown: suckling, feeding, and maternal contact in the absence of feeding all appear as good candidates. Contact with an anesthetized female has been shown to suppress the response to novelty in pups previously deprived of maternal contact (Stanton \& Levine, 1988, 1990; Stanton et al., 1987). Thus, maternal contact, per se, in the absence of feeding has been shown to regulate at least some aspect of the pup's HPA responsivity during the SHRP. Kuhn et al. (1990) have shown that the increase in basal levels of CORT seen following $2 \mathrm{~h}$ of maternal deprivation in 10-day-old pups is partially reversed when the pup is allowed to contact a nipple-cauterized dam. In the present experiment, we examined the role of maternal contact in the absence of suckling or feeding over a 24-h period. Specifically, Experiment 2 asked whether or not thermotactile contact with the dam contributes to the hyporesponsivity of the rat pup's HPA system. Thus, 8-, 12-, and 16-day-old pups were maternally deprived, left for $24 \mathrm{~h}$ with a female that exhibited maternal care but was unable to deliver milk (i.e., thelectomized dam), or left with their lactating mothers. At the end of this period, basal or stress (novelty) levels of CORT were compared between the different treatments.

\section{Method}

\section{Procedure}

Deprivation. Pups were taken from 12 different litters at each age. Litters were randomly assigned to one of the following pretesting conditions: pups were deprived with their littermates $(\mathbf{M}-\mathbf{L}+)$ or were left in the presence of either a thelectomized female (THEL) or their own mother $(\mathrm{M}+\mathrm{L}+)$. In the THEL condition, the mother was replaced by a thelectomized female $24 \mathrm{~h}$ prior to testing. These fethales were multiparous dams whose nipples had been surgically removed under ether anesthesia prior to parturition. Following delivery of their own pups, the thelectomized females were assessed regularly for presence of maternal behavior and absence of milk flow. Maternal behavior was maintained in these dams by providing them with pups from lactating "donor" females and rotating the infants between the thelectomized females and the donor mothers (these donor litters were not used in the experiment).

Testing. On the day of testing (i.e., Postnatal Days 8, 12, and 16), each of the litters in each deprivation condition and age group underwent equivalent testing procedures, as in Experiment 1: half of the animals were sacrificed immediately (NT), and the remainder were placed in a novel environment and sacrificed $30 \mathrm{~min}$ later (NOV). 

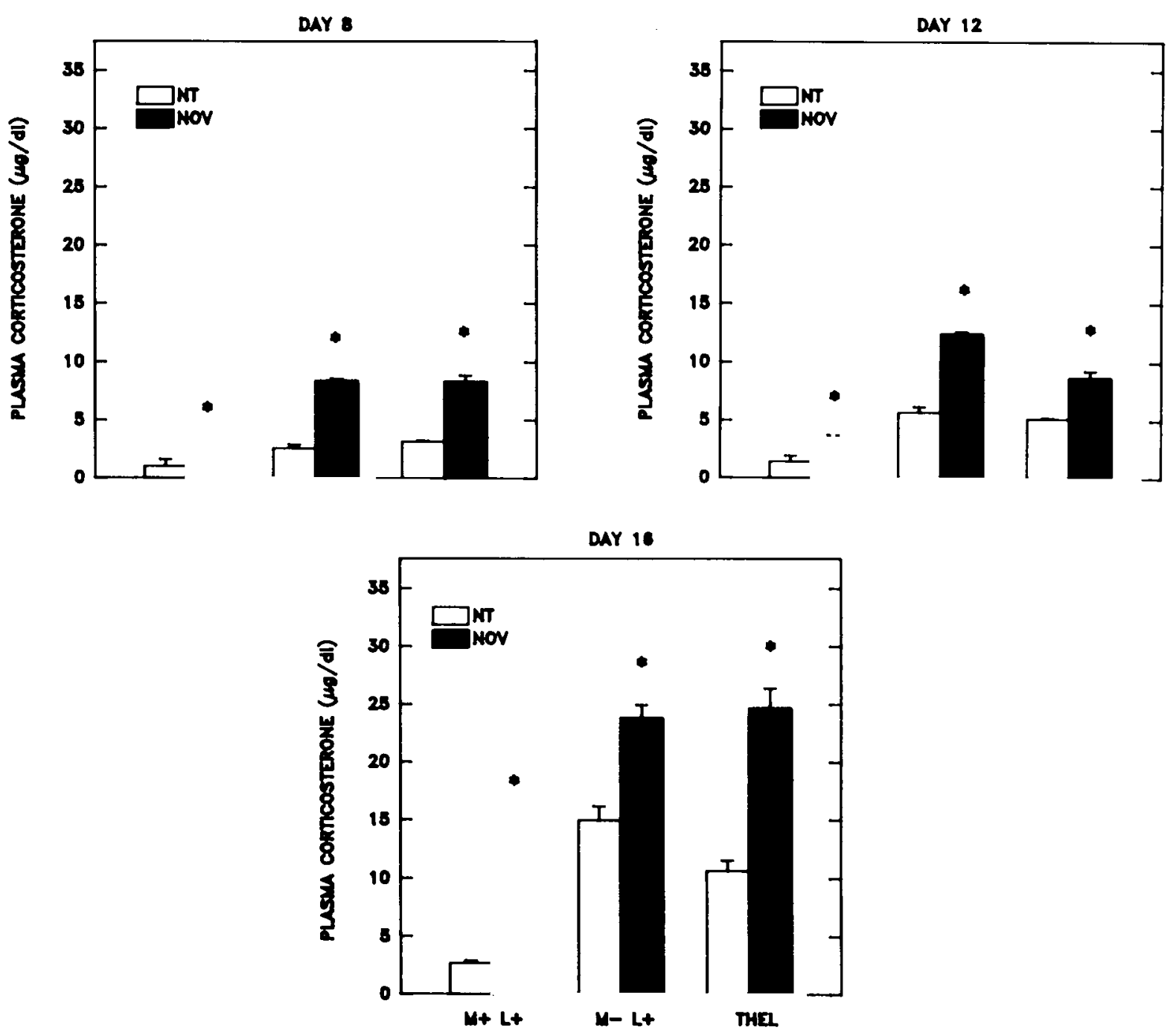

Figure 2. Plasma corticosterone levels $(\mu \mathrm{g} / \mathrm{d})$ in rat pups at different ages, under the sampling conditions of Experiment 2 . During the $\mathbf{2 4}$ h prior to testing, pups were left with their mothers $(M+L+)$, maternally deprived with their littermates $(M-L+)$, or left in the presence of a thelectomized female (THEL). At the time of testing, they were either sacrificed immediately (nontreated, or NT) or placed individually in a novelty test chamber for 30 min and then blood-sampled at the end of this period (novelty, or NOV). Values correspond to the mean $( \pm S E M)$. Within each group, values statistically greater than NT are marked with an asterisk. For intergroup post hoc comparisons, refer to the Results section in the text.

\section{Results}

The results of Experiment 2 are shown in Figure 2. Data were analyzed separately at each age by means of a 3 (condition, $M-L+$ vs. THEL vs. $M+L+) \times 2$ (treatment, NT vs. NOV) $\times 2$ (sex, male vs. female) ANOVA. Once it was determined that sex was not a significant factor, the data were collapsed across this variable and analyzed by means of a 3 (condition) $\times 2$ (treatment) ANOVA. No interactions due to litter effects were found between condition and treatment; therefore, individual values from each litter were used ( $n=16$ for each cell).

\section{Postnatal Day 8}

An ANOVA revealed an interaction between condition and treatment $[F(2,90)=22.97, p<.001]$. NT CORT levels in THEL pups were significantly elevated over $\mathrm{M}+\mathrm{L}+$ animals, whereas no difference was found between THEL and $\mathbf{M}-\mathbf{L}+$ basal CORT values. Although all groups showed a CORT response to novelty, this response was far greater in $\mathrm{M}-\mathrm{L}+$ and THEL pups. NOV CORT levels were significantly elevated over those of $M+L+$ pups in both THEL and $\mathrm{M}-\mathrm{L}+$ groups. Thus, at this age, passive contact with a thelectomized female had the same effect as total deprivation on NOV CORT levels (i.e., both conditions resulted in a potentiated CORT response to stress, as relative to that of nondeprived pups).

\section{Postnatal Day 12}

An ANOVA revealed an interaction between condition and treatment $[F(2,90)=18.29, p<.001]$. A Newman- 
Keuls analysis showed that both $\mathrm{M}-\mathrm{L}+$ and THEL pups had higher NT CORT levels than did M+L+ pups and did not differ from each other. Exposure to novelty led to an increase in CORT concentrations above NT levels in all conditions. NOV CORT levels of $M-L+$ pups were significantly higher than those of THEL pups, which, in turn, were higher than those of $\mathrm{M}+\mathrm{L}+$ pups. These results suggest that, at this age, contact with a nonlactating female is able to maintain the pup's HPA system in a partially suppressed state. It is evident, however, that a thelectomized female is not as effective as a normal mother is in inhibiting the infant's adrenocortical activity: NOV levels of CORT in THEL pups, although lower than those found in maternally deprived pups $(\mathbf{M}-\mathbf{L}+)$, were still significantly higher than those of $M+L+$ pups. Furthermore, contact with a thelectomized female did not maintain NT CORT secretion at M+L+ levels.

\section{Postnatal Day 16}

An ANOVA revealed an interaction between condition and treatment $[F(2,90)=2.98, p<.05]$. THEL pups showed NT levels of CORT that were lower than those found in $\mathrm{M}-\mathrm{L}+$ infants but were higher than those of $\mathrm{M}+\mathrm{L}+$ pups. Thus, at this age, the thelectomized female did reduce the pup's NT CORT secretion, although not to the same degree as an intact mother. All conditions showed significantly elevated NOV levels of CORT, relative to NT levels. This elevation was of greater magnitude than that found in 12-day-old pups, reflecting the fact that, by this age, pups are emerging from their hyporesponsivity and are thus capable, even when nondeprived, of showing a stress response. Contact with a thelectomized dam had no effect on stress-induced CORT secretion: both $M-L+$ and THEL pups showed significantly higher NOV CORT levels than did $\mathrm{M}+\mathrm{L}+$ pups and did not differ from each other.

Overall, these results show that contact with a thelectomized female was not sufficient to maintain the infant's HPA axis in its normal hyporesponsive state. It was, however, partially effective in reducing stress-induced CORT secretion in 12-day-old animals and basal CORT levels in 16-day-old pups.

\section{EXPERIMENT 3}

In Experiments 1 and 2, we have shown that the effect of deprivation on the developing HPA system is not related to contact with littermates and that it is only partially related to maternal contact. As stated in the introduction, the SHRP has been proposed to be a consequence of an enhanced negative feedback signal of CORT that would result in a suppression of ACTH release (Sakly \& Koch, 1983; Schapiro, 1965; Walker et al., 1986). Small amounts of maternal CORT are transferred to the neonate through milk (Zarrow, Philpott, \& Denenberg, 1970). If the hyporesponsiveness of the HPA system during ontogeny is indeed due to an enhanced feedback action of
CORT, then it is possible that even the small amounts of CORT present in the milk may contribute to this signal, resulting in a more pronounced suppression of the infant's HPA system. In order to test this hypothesis, in Experiment 3, maternally deprived pups were compared with pups allowed to remain with either an adrenalectomized (ADX) dam or with an intact dam. As in the previous two experiments, the CORT response to novelty was used as one index of HPA activity. Although we assume that the effects of maternal deprivation are mediated by changes in the neuroendocrine regulation at the central nervous system level, it is also apparent that the adrenal gland of the deprived infants is rendered more sensitive to exogenous and probably endogenous ACTH (Rosenfeld \& Levine, in press). There is an extensive literature that indicates that many of the functions of the adrenal are regulated by ACTH (Ramachandran, Jagannadha Rao, \& Liles, 1977). During the SHRP, circulating levels of ACTH are very low and stable in the infant (Guillet \& Michaelson, 1978). These low levels may in part be responsible for the relative hyporesponsiveness of the adrenal during this period. If maintenance of these low levels of ACTH is a consequence of the hypothesized enhanced negative feedback, and if glucocorticoids of maternal origin contribute to the feedback regulation, we would predict that pups reared with ADX mothers would be more responsive to exogenous $\mathrm{ACTH}$. This would be due to an increase in circulating ACTH as a result of the reduced negative feedback signal in the absence of maternal CORT. In order to test this hypothesis, two additional groups were tested in Experiment 3. One group was injected with ACTH, and another group was injected with saline as a control for the injection procedure.

\section{Method}

\section{Procedure}

Deprivation. Animals were taken from 15 litters at each age. Litters were randomly assigned to one of the following pretesting conditions: pups were deprived with their littermates $(M-L+)$, left in the presence of an ADX female (BDEP), or cross-fostered to an intact lactating female $(\mathrm{M}+\mathrm{L}+)$. The mothers of pups in the BDEP condition were removed from the cage and replaced for $24 \mathrm{~h}$ by a female whose adrenal glands had been surgically removed before breeding. ADX females show normal maternal behavior but have no CORT in milk. $\mathrm{M}+\mathrm{L}+$ pups were left undisturbed in their home cage until $24 \mathrm{~h}$ prior to testing, at which point they were crossfostered to an intact lactating female (this group therefore underwent the same procedures as the BDEP pups).

Testing. On the day of testing (i.e., Postnatal Days 8 and 12), each of the litters in each deprivation condition and age group underwent equivalent testing procedures: 2 pups ( 1 male and $1 \mathrm{fe}$ male) from each litter were weighed and then blood-sampled immediately (NT). The remaining 6 pups were randomly assigned to one of the following three treatments: (1) 2 pups (1 male and 1 female) were placed individually in a novelty chamber for $30 \mathrm{~min}$ (NOV); (2) 2 pups (1 male and 1 female) were given an i.p. injection of saline $(0.9 \% \mathrm{NaCl}$, in a volume of $1 \%$ body weight) and then placed in a novelty chamber for $30 \mathrm{~min}$ (SAL); (3) 2 pups (1 male and 1 female) were given an i.p. injection (1 IU/100 gbw in a volume of $1 \%$ body weight) of $\mathrm{ACTH}_{1-24}$ (Cortrosyn, Organon 
Inc., West Orange, NJ) and then placed in a novelty chamber for $30 \mathrm{~min}$ (ACTH). At the end of the $30 \mathrm{~min}$, blood was collected and assayed for CORT as described in General Methods.

Surgery. ADX was performed by the dorsal approach under ether anesthesia at least 7 days before breeding. Salt licks were provided after surgery to prevent electrolytic imbalance. Approximately 6 days before breeding, and until Day 14 of pregnancy, corticosterone (Steraloids, Inc., Wilton, NH 03086) was dissolved in alcohol and administered in the drinking water $(40 \mathrm{pg} / \mathrm{ml})$. This CORT replacement was used to ensure successful pregnancies.

\section{Results}

The results of Experiment 3 are shown in Figure 3. Data were analyzed separately at each age by means of a 3 (condition, $M-L+$ vs. BDEP vs. $M+L+$ ) $\times 4$ (treatment, $N T$ vs. NOV vs. SAL vs. ACTH) $\times 2$ (sex, male vs. female) ANOVA. Once it was determined that sex was not a significant factor, the data were collapsed across this variable and analyzed by means of a 3 (condition) $\times 4$ (treatment) ANOVA. No interactions due to litter effects were found between the conditions and the treatments; therefore, individual values from each litter were used $(n=$ 30 for each cell).

\section{Postnatal Day 8}

An ANOVA revealed main effects of condition $[F(2,106)$ $=48.61, p<.001]$ and treatment $[F(3,106)=13.80$, $p<.001] . \mathrm{M}-\mathrm{L}+$ pups showed a significant CORT response to novelty, saline and ACTH; this response did not differ between treatments. In contrast, neither BDEP nor $M+L+$ pups showed a CORT response to any of the treatments, with the exception of a small but significant CORT elevation in ACTH-treated $\mathrm{M}+\mathrm{L}+$ pups. Thus, at this age, deprivation rendered the pup responsive to novelty, saline, or ACTH injection. The absence of CORT in the milk had no effect on the pup's responsiveness, as evidenced by the similar patterns of response found in both BDEP and $\mathrm{M}+\mathrm{L}+$ pups.

\section{Postnatal Day 12}

An ANOVA revealed main effects of condition $[F(2,108)$ $=52.06, p<.001]$ and treatment $[F(3,108)=10.94$, $p<.001] . \mathrm{M}-\mathrm{L}+$ pups showed a pattern of response to the different treatments that resembled the one found at 8 days of age. At 12 days of age, however, the pups exhibited a far more pronounced adrenocortical response to the different treatments, as well as higher NT levels. Interestingly, in the BDEP (but not in the $\mathrm{M}+\mathrm{L}+$ ) condition, both saline and ACTH injection resulted in a significant elevation of CORT levels above NT values. This elevation, however, was significantly smaller than that observed in $\mathbf{M}-\mathbf{L}+$ pups. This suggests that, at this later age, an ADX mother is not as effective as an intact mother in inhibiting the pup's adrenocortical secretion.

Overall, the results of Experiment 3 show that the absence of maternal GCs has little effect on the hyporesponsiveness of the pup's HPA system. At 8 days of age, pups in the BDEP and $\mathrm{M}+\mathrm{L}+$ conditions showed similar CORT levels. In 12-day-old pups, however, saline or ACTH in-
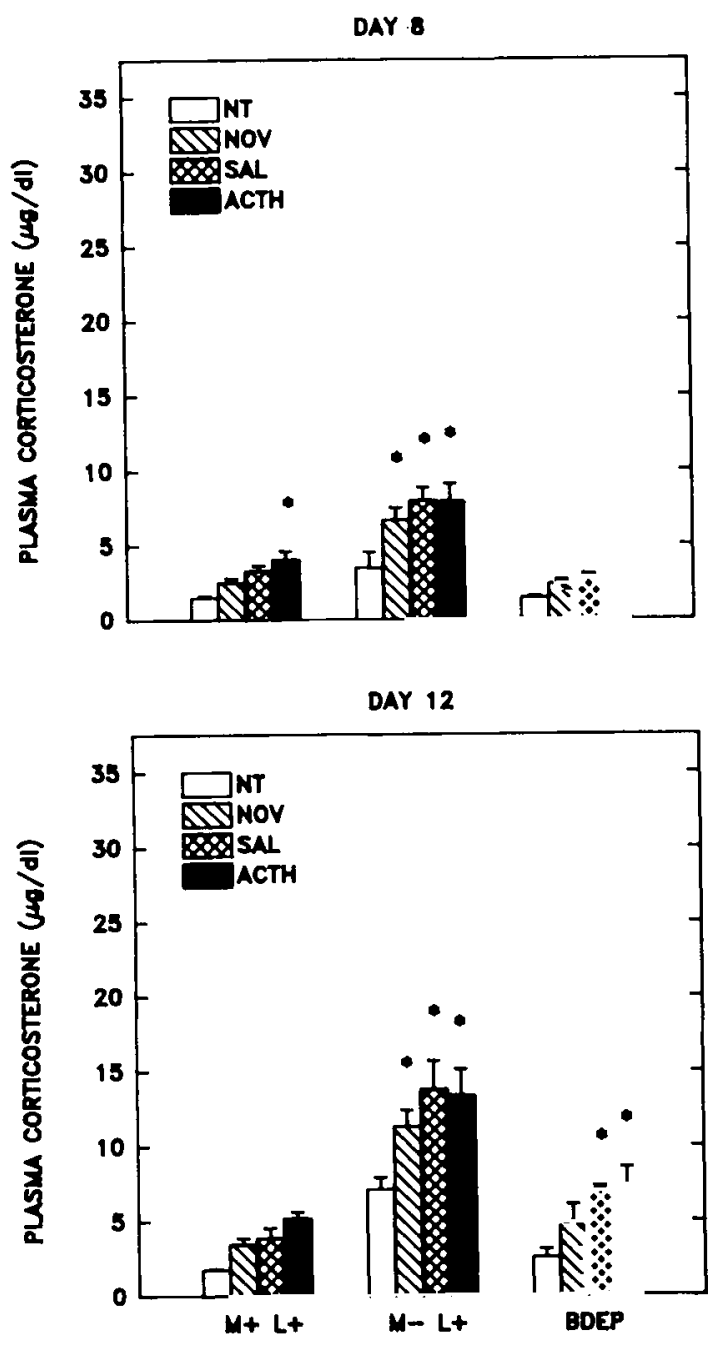

Figure 3. Plasma corticosterone levels $(\mu g / d)$ in rat pups at different ages, under the sampling conditions of Experiment 3. During the $\mathbf{2 4} \mathrm{h}$ prior to testing, pups were left with a lactating female $(\mathbf{M}+\mathbf{L}+)$, maternally deprived with their littermates $(\mathbf{M}-\mathbf{L}+)$, or left in the presence of an adrenalectomized lactating female (BDEP). At the time of testing, they were sacrificed immediately (nontreated, or NT), placed individually in a novelty test chamber for $30 \mathrm{~min}$, and then blood-sampled at the end of this period (novelty, or NOV), or injected with either saline (SAL) or ACTH (ACTH), placed in a novelty chamber for $30 \mathrm{~min}$, and then blood-sampled at the end of this period. Values correspond to the mean $( \pm S E M)$. Within each group, values statistically greater than NT are marked with an asterisk. For further intragroup (i.e., ACTH vs. SAL vs. NOV) and intergroup post hoc comparisons, refer to the Results section in the text.

jection, followed by novelty exposure, did elicit a small, albeit significant, adrenocortical response in BDEP, though not in $\mathrm{M}+\mathrm{L}+$ infants.

\section{GENERAL DISCUSSION}

The results of these experiments indicate that the major effects of deprivation on the developing HPA system are specifically due to the lack of maternal presence, rather 
than to the absence of social interactions. The deprivation effects are only partially prevented by maternal contact in the absence of feeding, and, furthermore, they are not substantially affected by the presence or absence of CORT in the mother's milk.

In Experiment 1, pups deprived of their mothers showed a significant increase in circulating CORT levels; this was true whether they had been maintained as a litter or they had been totally isolated. In both cases, these infants were different from pups reared with their mothers. Although the availability of littermates has been shown to influence other important biological functions (e.g., temperature regulation; Alberts, 1978), access to littermates does not appear to exert a role resembling that of the mother in regulating the HPA system. The results of Experiment 1 suggest that the effectiveness of the mother in modulating the pup's endocrine response to stress is not a function of some process related to social affiliation; rather, it appears to be dependent on some aspect of maternal behavior.

The data from Experiment 2 provide only little evidence that maternal contact in the absence of suckling and/or feeding is capable of downregulating the HPA system. Thus, pups that remained with thelectomized mothers showed CORT levels equivalent to those of maternally deprived infants (with the exception of a small but significant modulation of the CORT response at Postnatal Day 12); in all cases, these levels were significantly higher than those of nondeprived pups. Pups in contact with nipple-removed dams cannot feed or suckle. Because thelectomized females have been described not to exhibit an active lactating posture, infants in contact with these dams also did not experience this particular aspect of normal maternal behavior (Stern, 1990). The data from Experiment 2 do not support the observations of Kuhn et al. (1990). These investigators reported that pups reared with nipple-cauterized dams showed a reduction in their CORT levels, relative to that of maternally deprived infants. However, these CORT values were still significantly higher than those of control pups. Again, it should be noted that in terms of absolute magnitude of circulating CORT the values are approximately $1 \mu \mathrm{g} / \mathrm{dl}$. It is difficult to compare the results of Experiment 2 with those obtained by Kuhn et al. (1990). In the experiments presented in this paper, the pups were deprived for $24 \mathrm{~h}$, as opposed to $2 \mathrm{~h}$ of deprivation used by these authors. However, we did not observe any reduction of the elevations in basal CORT levels that are common in pups that are maternally deprived for $24 \mathrm{~h}$ at 8 and 12 days of age. In 16-day-old pups reared with thelectomized mothers, a slight but significant reduction in basal levels was noticed. The magnitude of these elevations were age dependent and tended to increase with age. It is conceivable that the effects of nonnutritional maternal contact may be apparent during the early phase of the deprivation period, but they are no longer manifest after prolonged deprivation.

These data were surprising since it has been shown that even an anesthetized mother is a potent inhibitor of the (24-h maternally deprived) infant's CORT response to novelty (Stanton \& Levine, 1988; Stanton et al., 1987). In this case, the inhibition is a result of contact per se between the mother and infant (Stanton \& Levine, 1990). In Experiment 2, however, pups allowed to make contact with a dam, but not to suckle or ingest milk, did not differ from maternally deprived pups. This suggests that the processes responsible for maintaining the SHRP differ from those that modulate the stress response in an infant that has been rendered responsive by $24 \mathrm{~h}$ of maternal deprivation. Thus, we can discriminate two independent events: (1) an inhibiting effect that maintains hyporesponsivity, and (2) a suppressing effect that prevents the CORT response from being activated when the infant is capable of responding to mildly stressful stimuli. The latter is a function of contact; however, the mechanism involved in the former has not as yet been elucidated. Contact per se, however, although capable of modulating the hyporesponsiveness of the infant's HPA axis, is not the critical variable in maintaining this system in its basal, unstimulated state.

One of the mechanisms proposed to explain the hyporesponsivity of the HPA system during the SHRP is that, during this period, there is an enhanced CORT negative feedback signal (Sakly \& Koch, 1983; Schapiro, 1965; Walker et al., 1986). This enhanced signal would result from a combination of developmental factors. First, adultlike concentrations of glucocorticoid receptors (presumed to mediate the feedback effects of CORT) are present in the pituitary at the time of birth and remain at constant levels throughout development (Sakly \& Koch, 1981). Second, corticosterone-binding globulin (CBG) levels are markedly reduced during the SHRP (Henning, 1978). Thus, CORT would exist primarily in the unbound (i.e., biologically active) form. In consequence, even "low" levels of CORT could provide a potent negative feedback signal, which could not be overridden by corticotropin releasing factor. Even the low amount of CORT present in the mother's milk might therefore be hypothesized to contribute to the enhancement of the negative feedback action of CORT.

The results of Experiment 3, however, do not support the above hypothesis. Eight-day-old pups reared with ADX mothers for the $24 \mathrm{~h}$ prior to testing were as nonresponsive as pups reared with intact mothers. On Postnatal Day 12, the CORT-deprived infants were slightly more responsive than the control nondeprived pups to both saline and ACTH injections; however, the response was markedly smaller than that found in deprived pups.

Although we cannot dismiss an age-related CORT effect, there is an alternative explanation that is more consistent with the data presented in Experiments 1 and 2 . Thus far, all the data presented suggest that the critical aspect of the mother-infant interaction responsible for maintaining the SHRP is related to the feeding process, either milk, suckling, or both. ADX lactating females have been shown to exhibit normal maternal behavior. Circulating CORT, however, is required for the production of normal quantities of milk: in the absence of CORT, less 
milk is produced (Thoman, Sproul, Seeler, \& Levine, 1970). The amount of milk supplied by the ADX dams might therefore be sufficient to satisfy the relatively small nutritional demands of Postnatal Day 8 pups but not of older (i.e., Postnatal Day 12) pups, which have greater nutritional demands. If milk intake is indeed the critical factor, then the reduction of milk intake in the 12-dayold animals might be sufficient to explain the partial disinhibition of the HPA system seen at this age.

Overall, these data support the hypothesis that the ontogeny of the HPA system is another developmental process that is partially under maternal regulation. The presence of the mother appears to inhibit the pup's HPA system, maintaining it hyporesponsive to external stimuli and reducing the sensitivity of the adrenal to exogenous ACTH. These results suggest that one of the critical factors that may be responsible for maternal downregulation of the neonatal HPA system may be related to feeding. In the adult rat, feeding has been shown to suppress the HPA axis (Dallman, 1984; Levine, 1986). This hypothesis was tested in a recent study (Rosenfeld, Ekstrand, Olson, \& Levine, in press), in which 12-day-old pups were deprived of their mothers but were fed over a 24-h period through a cheek cannula. The amount of food delivered was sufficient to produce the weight gain observed in maternally reared pups. In these animals, basal levels of CORT were equivalent to the maternally reared pups. However, although feeding did markedly reduce the response to saline injection, it did not completely eliminate it. It appears therefore that maternal suppression of the HPA axis during the SHRP is a function of a combination of maternal behaviors that act in concert to maintain the infant in a hyporesponsive state.

\section{REFERENCES}

AlberTs, J. R. (1978). Huddling by rat pups: Group behavioral mechanisms of temperature regulation and energy conservation. Journal of Comparative \& Physiological Psychology, 92, 231-245.

Dallman, M. F. (1984). Viewing the ventromedial hypothalamus from the adrenal gland. American Journal of Physiology, 246, R1-R12.

De Kloet, E. R., Rosenfeld, P., Van Eekelen, J. A. M., Sutanto, W., Levine, S. (1988). Stress, glucocorticoids and development. In G. J. Boer, M. G. P. Feenstra, M. Mirmiran, \& D. F. Swaab (Eds.), Progress in brain research (pp. 101-120). Amsterdam: Elsevier Science.

Evoniuk, G. E., Kuhn, C. M., Schanberg, S. M. (1979). The effect of tactile stimulation on serum growth hormone and tissue ornithine docarboxylase activity during maternal deprivation in rat pups. Communications in Psychophamacology, 3, 363-370.

Guillet, R., Michaelson, S. M. (1978). Corticotropin responsiveness in the neonatal rat. Neuroendocrinology, 27, 119-125.

HenNing, S. J. (1978). Plasma concentrations of total and free corticosterone during development in the rat. American Joumal of Physiology, 235, E451-E456.

HoFer, M. A. (1973). The role of nutrition in the physiological and behavioral effects of early maternal separation on infant rats. Psychosomatic Medicine, 35, 350-359.

HoFer, M. A. (1987). Shaping forces within early social relationships. In N. A. Krasnegor, E. M. Blass, M. A. Hofer, \& W. P. Smotherman (Eds.), Perinatal development: A psychobiological perspective (pp. 251-274). New York: Academic Press.
HoFer, M. A., SHAIR, H. (1978). Ultrasonic vocalization during social interaction and isolation in 2-week-old rats. Developmental Psychobiology, 11, 495-504.

HoFer, M. A., Shair, H. (1980). Sensory processes in control of isolation-induced ultrasonic vocalization by 2 -week-old rats. Joumal of Comparative \& Physiological Psychology, 94, 271-279.

Hofer, M. A., Shair, H. (1982). Control of sleep-wake states in the infant rat by features of the mother-infant relationship. Developmental Psychobiology, 15, 229-243.

KLemm, W., \& GuptA, D. (1975). A routine method for the radioimmunoassay of plasma cortisol without chromatography. In D. Gupta (Ed.), Radioimmunoassay of steroid hormones (pp. 143-151). Weinheim, Germany: Verlag Chemie.

Kuhn, C. M., But ler, S. R., \&Chanberg, S. M. (1978). Selective depression of serum growth hormone during maternal deprivation in rat pups. Science, 201, 1034-1036.

Kuhn, C. M., Pauk, J., \& SChanberg, S. M. (1990). Endocrine responses to mother-infant separation in developing rats. Developmental Psychobiology, 23, 395-410.

Levine, S. (1986). Hormones and feeding: Cause and effect. In E. Ferrari \& F. Brambilla (Eds.), Disorders of eating behaviour (pp. 73. 84). New York: Pergamon Press.

LeVIne, S., Huchton, D. M., WIENER, S. G., Rosenfeld P. (1991) Time course of the effect of maternal deprivation on the hypothalamicpiruitary-adrenal axis in the infant rat. Developmental Psychobiology, 24, 547-558

Ramachandran, J., Jagannadha RAo, A., Liles, S. (1977). Studies of the trophic action of ACTH. Annals of the New York Academy of Sciences, 297, 336-348.

Rosenfeld, P., Exstrand, J., Olson, E., Levine, S. (in press) Maternal regulation of hypothalamic-pituitary-adrenal activity in the infant rat: Effects of feeding. Developmental Psychobiology.

Rosenfeld, P., Gutierrez, Y. A., Martin, A. M., Mallett, H. A., Alleva, E., \&eVine, S. (1991). Maternal regulation of the adrenocortical response in preweanling rats. Physiology \& Behavior, 50, 661-671.

ROSENFELD, P., \&EVINE, S. (in press). Maternal deprivation increases adrenal sensitivity to adrenocorticotropin. Joumal of Endocrinology.

SAKLY, M., KoCH, B. (1981). Ontogenesis of glucocorticoid receptors in anterior pituitary gland: Transient dissociation among cytoplasmic receptor density, nuclear uptake, and regulation of corticotropic activity. Endocrinology, 108, 591-596.

SAKLY, M., \& КоСH, B. (1983). Ontogenetical variations of transcortin modulate glucocorticoid receptor function and corticotropic activity in the pituitary gland. Hormones \& Metabolism Research, 15 , 92-96.

SAPOLSKY, R. M., MEANEY, M. J. (1986). Maturation of the adrenocortical stress response: Neuroendocrine control mochanisms and the stress hyporesponsive period. Brain Research Reviews, 11, 65-76.

SCHAPiRo, S. (1965). Neonatal cortisol administration: Effect on growth, the adrenal gland and pituitary-adrenal response to stress. Joumal of Pharmacological \& Experimental Therapy, 139, 771-774.

Stanton, M. E., Gutierrez, Y. R., \& Levine, S. (1988). Maternal deprivation potentiates pituitary-adrenal stress responses in infant rats. Behavioral Neuroscience, 102, 692-700.

Stanton, M. E., \& Levine, S. (1988). Maternal modulation of infant glucocorticoid stress responses: Role of age and maternal deprivation. Psychobiology, 16, 223-228.

Stanton, M. E., Levine, S. (1990). Inhibition of infant glucocorticoid stress response: Specific role of maternal cues. Developmental Psychobiology, 23, 411-426.

Stanton, M. E., Wallstrom, J., Levine, S. (1987). Maternal contact inhibits pituitary-adrenal stress responses in preweanling rats. Developmental Psychobiology, 20, 131-145.

STERN, J. (1990, July). Sensory and dopaminergic regulation of maternal behavior in Nonway rats: Critical role of trigeminal and ventral somatosensory inputs (Abstract). Paper presented at the Annual Meeting of the International Society for Developmental Psychobiology, Cambridge, U.K. 
Thoman, E. B., Sproul, M., Seeler, B., \& Levine S. (1970). Influence of adrenalectomy in female rats on reproductive processes including effects on the foetus and offspring. Journal of Endocrinology, 46, 297-303.

Walker, C. D., Sapolsky, R. M., Meaney, M. J., Vale, W. W., \& Rivier, C. L. (1986). Increased pituitary sensitivity to glucocorticoid feedback during the stress nonresponsive period in the neonatal rat. Endocrinology, 119, 1816-1821.

WIDMAIER, E. P. (1990). Glucose homeostasis and hypothalamicpituitary-adrenocortical axis during development in rats. American Journal of Physiology, 259, E601-E613.
WINER, B. J. (1971). Statistical principles in experimental design (2nd ed.). New York: McGraw-Hill.

Zarrow, M. X., Philpott, J. E., Denenberg, V. H. (1970). Passage of $14 \mathrm{C}-4$-corticosterone from the rat mother to the foetus and neonate. Nature, 226, 1058-1059.

(Manuscript received August 26, 1991; revision accepted for publication February 25,1992 .) 